\title{
El principio de autonomía e independencia en la declaración sobre la identidad cooperativa
}

(The principle of autonomy and independence in the declaration on cooperative identity)

\author{
Dante Cracogna ${ }^{1}$ \\ Universidad de Buenos Aires (Argentina)
}

Sumario: I. Significado del principio. II. Heterogeneidad del contenido. III. La independencia en perspectiva histórica. IV. El papel del Estado. Nuevos enfoques. V. Panorama previo al Congreso del Centenario. VI. Desafíos no estatales. La necesidad de capital. VII. El gobierno corporativo. VIII. Supervisión y control. IX. Recaudos legales y constitucionales. El caso del Mercosur. X. Conclusión. XI. Bibliografía.

Summary: I. Meaning of the principle. II. Complexity of the content. III. Historical prospective of independence. IV. Rol of the State. New visions. V. Scenary previous to the Centennial Congress. VI. Non State challenges. The need of capital. VII. Corporate governance. VIII. Supervision and control. IX. Legal and constitutional requirements. The Mercosur case. X. Conclusion. XI. Bibliography.

Resumen: Después de un análisis del significado y novedad del principio, el artículo destaca su doble perfil: autonomía al interior de la cooperativa e independencia respecto de agentes externos, básicamente los gobiernos y los inversores de capital. Hace un repaso histórico del tema de la relación de las cooperativas con el Estado hasta llegar al pronunciamiento del Congreso del Centenario en el que también se incorpora la cuestión relativa al capital externo (socios no usuarios) y su posible impacto sobre la autonomía y la independencia de las cooperativas. A continuación, trata otros aspectos susceptibles de afectar a las cooperativas en esta materia, tales como el gobierno corporativo y la supervisión estatal. Finaliza destacando la importancia de los recaudos legales para preservar los rasgos de las cooperativas, analizando la situación del tema en los países del Mercosur.

Palabras clave: autonomía, independencia, relaciones con el Estado, capital.

1 E-mail: dcracogna@estudiocracogna.com.ar 
Abstract: After considering the meaning and novelty of the principle, the article points out its double profile: autonomy at the interior of the cooperative and independence from external agents, mainly governments and capital investors. It then goes into a historical review of the relationship between cooperatives and the State up the outcome of the ICA Centennial Congress in which the issue of the external capital (investor members) was also introduced in connection with the autonomy and independence of cooperatives. Afterwards, other aspects which can affect their independence such as the corporate governance and the State supervision are considered. The article ends stressing the importance of the constitutional and legal provisions in order to ensure the particular features of the cooperatives making a reference to the situation in the Mercosur countries.

Keywords: autonomy, independence, relationship with the State, capital. 


\section{Significado del principio}

El 4. ${ }^{\circ}$ principio de la Declaración sobre la Identidad Cooperativa aprobada por la Alianza Cooperativa Internacional (ACI) en su Congreso del Centenario celebrado en Manchester en 1995 establece: «Las cooperativas son organizaciones autónomas de autoayuda gestionadas por sus miembros. Si establecen acuerdos con otras organizaciones -incluidos los gobiernos - o si reciben capital de fuentes externas, lo hacen en condiciones que garanticen el control democrático por parte de sus miembros y respeten su autonomía cooperativa.»

A diferencia de lo que sucede con los restantes principios, éste tiene la singularidad de que no reconoce antecedente expreso en las anteriores formulaciones de la $\mathrm{ACl}$. En efecto, puede decirse que los demás principios - excepto el $7 .^{\circ}$ - reexpresan el contenido de los que se hallaban contenidos en las declaraciones aprobadas por los congresos de París 1937 y de Viena 1966, en tanto que el $4 .^{\circ}$ - como el 7. - - están dotados de cierta originalidad. Ello no obsta a que puedan encontrarse raíces de sus respectivos contenidos en algunos de los principios anteriores.

De manera que este principio, aunque sus fundamentos puedan conectarse con significados implícitos en las formulaciones previas, adquiere a partir de 1995 una categórica y relevante presencia que lo proyecta a un plano totalmente equiparable con los restantes. Los principios de gobierno democrático y de neutralidad política $\left(2 .^{\circ}\right.$ y $\left.7 .^{\circ}\right)$ contenidos en la Declaración de 1937 y los de adhesión abierta y voluntaria sin discriminación política y de organización democrática $\left(1 .^{\circ}\right.$ y $\left.2 .^{\circ}\right)$ de la Declaración de 1966, entrañan las nociones genéricas de autonomía e independencia ahora explicitadas en forma manifiesta en el $4 .^{\circ}$ principio. ${ }^{2}$

2 Sin embargo, es notable que en la Declaración del Congreso de Viena de 1966 se suprimiera el enunciado expreso de neutralidad política contenido en la Declaración de 1937. Seguramente el contexto internacional de esa época, con la división del mundo en dos grandes bloques y el auge de la Guerra Fría, contribuyen a explicar esa situación en la que el movimiento cooperativo internacional pugnaba por conservar la unidad. En palabras de la comisión redactora del informe que sirvió de base para la nueva formulación de los principios cooperativos: "De lo anterior se deduce claramente que la Comisión considera que no se puede ratificar el informe de 1937, dando la misma autoridad absoluta a la neutralidad como un principio. En ciertas circunstancias, la neutralidad es un derecho y una línea de conducta apropiada. Debe haber libertad a todos los niveles de la estructura cooperativa para que los socios individuales, las sociedades primarias, las organizaciones secundarias y las instituciones internacionales tomen, hacia los asuntos políticos, las actitudes que sean necesarias o más apropiadas a las circunstancias en cualquier época o lugar.» (Alianza Cooperativa Internacional, Nuevos enfoques de los principios cooperativos en el mundo, 7. ${ }^{a}$ ed., Ediciones Idelcoop, Rosario, 1987, p. 69). 


\section{Heterogeneidad del contenido}

Es dable advertir que el texto del actual $4 .^{\circ}$ principio, aunque con un acápite preciso — «autonomía e independencia»- abarca distintos aspectos englobados dentro de un mismo enunciado.

La primera oración constituye una reafirmación de lo que inicialmente establece la definición de cooperativa que encabeza la Declaración sobre la Identidad Cooperativa: organizaciones autónomas de autoayuda gestionadas democráticamente por sus miembros. Con ese enunciado se brinda el marco del contenido del principio a la vez que se resume su esencia. A continuación, después de esta afirmación inicial, el texto avanza en establecer los límites y recaudos que las cooperativas deben observar en sus relaciones con agentes externos: gobiernos, inversores y otras organizaciones en general. Es decir que, primeramente se afirma su autonomía de gobierno como entidades democráticas; esa es la fase interna de su naturaleza y a continuación, se expresa cómo la cooperativa asegura su independencia frente a los terceros, cualesquiera que éstos fueran. Esa es la doble faz del principio que, en su conjunción, manifiesta la naturaleza cooperativa: autonomía de su gobierno democrático e independencia en sus relaciones externas.

De tal suerte, la heterogeneidad del contenido de este principio debe entenderse conducente a un rasgo único: la cooperativa es una entidad al servicio de sus miembros - que son sus propietarios y gestores- que se gobierna democráticamente. Siendo así, resulta obvio que se trata de una entidad autónoma e independiente, que responde solamente a quienes la forman y a ningún otro factor. El principio lo destaca y enfatiza, pero, en rigor, no agrega un dato novedoso a los que la cooperativa exhibe en función de su definición y de los demás principios.

Queda entonces por justificar su existencia. Y aquí la historia de las cooperativas desempeña un papel relevante para brindar la respuesta.

\section{La independencia en perspectiva histórica}

Cuando surgieron las primeras cooperativas, de las que Rochdale constituye ejemplo paradigmático, lo hicieron como genuina expresión de la voluntad libre de sus integrantes. A ninguna de ellas se le habría ocurrido acudir al Estado para sostener su organización; al contrario, muy posiblemente habrán pensado cómo defenderse de un Estado que no tomaba en cuenta las necesidades de importantes sectores de 
la sociedad de los que ellas se nutrían y, en última instancia, consentía la situación de postergación económica y social en que sus miembros se encontraban, tomando partido en favor de quienes eran responsables de ese estado de cosas. De ninguna manera, pues, constituía opción viable entrar en acuerdo o negociación con gobierno alguno. ${ }^{3}$ Por lo tanto, desde ese punto de vista, la independencia se hallaba presupuesta, y nadie habría postulado la necesidad afirmarla mediante un principio o pauta para la acción.

Sin embargo, a comienzos del siglo xx las cooperativas comenzaron a aparecer en países coloniales como consecuencia de la acción promocional de los gobiernos metropolitanos y a partir de allí se inauguró lo que Hans-H. Münkner denomina el modelo «indo-británico de cooperativismo», plasmado en la legislación india de 1904. ${ }^{4}$ Surge entonces una inédita relación entre Estado y cooperativas; frente a la actitud prescindente de aquél, típica de los países europeos y de América del Norte, nace una actitud de colaboración, de fomento hacia las cooperativas que pasan a ser consideradas como coadyuvantes de los objetivos de progreso económico y social que el Estado persigue. Estado y cooperativas son socios en una actividad común, con todos los riesgos que dicha situación conlleva.

El reconocimiento de que las cooperativas propenden a la promoción económica y consiguiente mejoramiento de la calidad de vida de sus miembros hace que el Estado descubra en ellas agentes eficaces para contribuir al logro de su labor tutelar del bien común general, incorporando a importantes sectores de la sociedad a la actividad económica formal y facilitando su acceso a un nivel socio cultural superior con beneficio para ellos y para el conjunto de la población.

\section{El papel del Estado. Nuevos enfoques}

Ese fue el factor que motivó novedosas políticas públicas orientadas a promover las cooperativas, desconocidas hasta entonces. Tales

${ }^{3}$ Cabe recordar que por aquel tiempo en Inglaterra el movimiento cartista — que fue una de las opciones que los pioneros consideraron asumir antes de decidirse por emprender un camino nuevo y distinto- postulaba, precisamente, la modificación de la legislación electoral para posibilitar que representantes de los sectores populares pudieran acceder al Parlamento y desde allí procurar la modificación del statu quo.

4 Münkner, Hans-H., ha tratado el tema con amplitud en diversas oportunidades, pero la publicación más interesante sobre el tema es: Münkner, Hans-H. (Editor), 100 Years Co-operative Credit Societies Act India, 1904, ICA Regional Office for Asia and the Pacific, New Delhi, 2005. 
políticas, en su declarado propósito de apoyo llegaron a diverso grado de intervención, incluso a desconocer la autonomía de las cooperativas en favor de una injerencia estatal en su organización y gestión que asegurara una supuesta mayor eficiencia. Las facilidades de fomento en materia de crédito, acceso a mercados o exenciones tributarias, se constituían en razones que justificaban - como contrapartida - una notable intromisión en la vida de las cooperativas a fin de asegurar el éxito de tales medidas.

Esta situación se extendió paulatinamente - con mayor o menor intensidad - a todos los países en desarrollo; es decir, a lo que se dio en llamar el Tercer Mundo, incluso con el apoyo de organismos internacionales y de agencias de desarrollo de países industrializados. Las décadas posteriores a la Segunda Guerra fueron una época de notable auge de esta política, durante la cual se realizaron numerosas reuniones, seminarios y congresos dedicados a analizar y proponer las mejores prácticas gubernamentales para la promoción de las cooperativas y se publicaron incontables estudios teóricos e informes sobre experiencias realizadas en esta materia. ${ }^{5}$

A pesar de lo dicho, no fueron los países en desarrollo los que llevaron adelante una política de mayor avance sobre la autonomía de las cooperativas. En efecto, ellos sólo marcaron un jalón de significativa importancia en cuanto a los riesgos de una azarosa relación con el Estado que solía estar teñida de un aura de mutua colaboración, frecuentemente idealizada por la existencia de propósitos compartidos.

Pero donde se produjo un decidido avasallamiento de la independencia de las cooperativas fue en los llamados países socialistas en los cuales no hubo intervencionismo sino incorporación o absorción de las cooperativas dentro de la planificación estatal centralizada. Resultaba obvio que las cooperativas no podían existir como unidades económicas autónomas dentro del marco de una política económica rígidamente planificada; por lo tanto, debían subordinar su actuación a los

5 Un interesante resumen de los problemas y las posiciones que se plantearon en esta materia puede consultarse en Drimer, Alicia Kaplan de - Drimer, Bernardo, Las cooperativas. Fundamentos-Historia-Doctrina, 4a. ed. actualizada por Mirta Vuotto, Intercoop, Buenos Aires, 2017, p. 537 y ss. Por otra parte, una muestra del tema se encuentra en la publicación de los debates que tuvieron lugar en la reunión del Comité Central de la $\mathrm{ACl}$ realizada en Copenhagen en 1978, en la que también se incluye una representativa bibliografía de la época (ICA, Co-operatives and the State, London, 1980). Utiles reflexiones, además de valiosa información, sobre el tema pueden consultarse en el capítulo titulado «El estado y el cooperativismo» de Uribe Garzón, Carlos, Bases del cooperativismo, 5a. ed., Fondo Nacional Universitario, Bogotá, 2002, p. 495 y ss. 
dictados de dicha política, realizando su actividad exclusivamente en las áreas geográficas y sectores económicos asignados y cumpliendo estrictamente las metas establecidas por la planificación. Ello suponía, generalmente, que los directivos de las cooperativas fuesen funcionarios públicos. En suma, las cooperativas solamente constituían una pieza dentro del engranaje de la economía centralmente planificada.

\section{Panorama previo al Congreso del Centenario}

De allí que cuando tuvo lugar la tarea preparatoria del Congreso del Centenario de la $\mathrm{ACl}$ —hacia fines de la década de 1980 -6 el

6 Puede considerarse que dichos trabajos preparatorios tuvieron un destacado comienzo con la realización del $27 .{ }^{\circ}$ Congreso de la $\mathrm{ACl}$ realizado en Moscú en octubre de 1980, en el que se presentaron dos informes que sirvieron de base a las deliberaciones y que representaban, claramente, las diferentes concepciones acerca de las cooperativas en los países occidentales y en los países socialistas. El primero, titulado "Las Cooperativas en el año 2000» fue escrito por el canadiense Alexander F. Laidlaw, quien destaca que el fuerte apoyo de los gobiernos termina siendo un abrazo de oso para las cooperativas (p.147). El segundo, sin autor mencionado, fue presentado por las organizaciones centrales de cooperativas de la URSS, Hungría, Bulgaria, República Democrática de Alemania, Polonia y Checoslovaquia, bajo el título «Las cooperativas en los países socialistas en el año $2000 »$. El título del primer parágrafo de este informe define el rol de las cooperativas: "Las cooperativas, parte integrante del sistema político y económico de la sociedad socialista», el que se afirma: "Como empresa económica, las cooperativas, siendo una de las formas de la propiedad socialista, son una parte integrante del sistema económico de la sociedad; como organización social, siendo una forma específica de la democracia, son una parte integrante de su sistema político, del mecanismo de la democracia socialista.» (p. 154) (Laidlaw, Alex F., Las cooperativas en el año 2000, ACl Oficina Regional para Centroamérica y el Caribe, San José, s/f). Los otros trabajos que pueden considerarse fundamentales como antecedentes de la Declaración sobre la Identidad Cooperativa son el informe del presidente de la $\mathrm{ACl}$ presentado en el XXIX Congreso realizado en Estocolmo en 1988 titulado, » Cooperativas y valores básicos», en el que destaca la importancia de la independencia de las cooperativas (Marcus, Lars, Cooperativas y valores básicos, El Hogar Obrero, Buenos Aires, s/f, p. 6-7) y el informe de Sven Ake Böök presentado en el Congreso de la $\mathrm{ACl}$ realizado en Tokio en 1992 que abrió el tramo final de los estudios previos al Congreso del Centenario (Böök, Sven Ake, Valores cooperativos para un mundo en cambio, ACl Oficina Regional para Centro América y el Caribe, San José, 1992). Por fin, aunque se trata de un trabajo realizado en forma independiente, es decir por fuera de la $\mathrm{ACl}$, debe mencionarse el libro escrito por un ex director de ese organismo que tuvo significativa influencia en la elaboración de la Declaración sobre la Identidad Cooperativa: Watkins, W.P., Cooperative Principles Today and Tomorrow, Holyoake Books, Manchester, 1986. En todos estos documentos se subraya la relevancia de la autonomía y la independencia de las cooperativas, sea en forma directa y explícita o a través de la afirmación de valores y principios que las suponen. 
mapa del movimiento cooperativo mundial en punto a su relación con el Estado abarcaba tres diferentes regiones, cada una de ellas caracterizada por una diferente tendencia, a saber:

a) países industrializados: actitud indiferente o prescindente del Estado hacia las cooperativas, con inclinación a considerarlas como una forma más de organización empresaria;

b) países en desarrollo: el Estado asume una actitud de promoción de las cooperativas, considerándolas un instrumento de desarrollo económico y social coadyuvante a sus propias finalidades: y

c) países socialistas: el Estado incorpora a las cooperativas como parte de la economía centralmente planificada. ${ }^{7}$

De tal suerte, si bien la caída del Muro de Berlín producida a comienzos de la década siguiente y el comienzo de la globalización económica introdujeron una significativa novedad en el panorama mundial, la ACl estaba en cierta forma apremiada a pronunciarse acerca de un tema que las anteriores proclamaciones de principios sólo se hallaba insinuada: la independencia de las cooperativas con relación al Estado.

\section{Desafíos no estatales. La necesidad de capital}

Sin embargo, también la $\mathrm{ACl}$ debía hacerse cargo de situaciones que comprometen o amenazan la autonomía y la independencia de las cooperativas, especialmente en los países de economía desarrollada en los que por imperativo de su crecimiento ellas realizan acuerdos con empresas no cooperativas o bien deben acudir a la incorporación de capital de fuentes externas. ${ }^{8}$ En tales casos, las consecuencias de realizar esos acuerdos o captar esos capitales pueden significar el sacrificio de su independencia o la pérdida de autonomía en su gobierno. De allí que el principio haga referencia expresa también a estas situaciones en las que la amenaza no proviene del Estado sino de otras organizaciones del sector privado de la economía. Obviamente, el riesgo también invo-

7 Sobre las diferentes actitudes del Estado frente a las cooperativas traducida en la legislación cooperativa cfr. Cracogna, Dante, Estudios de Derecho Cooperativo, Intercoop, Buenos Aries, 1986, p. 261 y ss.

8 El Documento de referencia, redactado por lan Mac Pherson, que acompañó a la Declaración sobre la Identidad Cooperativa, puntualiza esta situación agregando «que no existe razón para creer que esta tendencia se revertirá.» (Alianza Cooperativa Internacional, Los principios cooperativos para el siglo XXI, Intercoop, Buenos Aires, 1996, p. 43). 
lucra a la asistencia financiera o técnica proveniente de entidades que, aunque no sean naturaleza empresaria, también por su índole o magnitud pueden llegar a condicionar la autonomía de las cooperativas.

Cabe señalar que en este cuarto principio se dio cabida expresa a una posibilidad que, aunque legalmente reconocida por algunas legislaciones ${ }^{9}$, había dado motivo a gran controversia en el mundo cooperativo: la posibilidad de que las cooperativas capten capital de terceros. Es decir, no solamente que se endeuden por vía de financiamiento externo sino que incorporen aportes de capital de socios no usuarios de sus servicios (los llamados «socios capitalistas»), con el consiguiente derecho a intervenir en las decisiones sociales y formar parte de los órganos de la cooperativa (asamblea y consejo de administración).

El tema de tales aportes de capital está tratado en este principio puesto que el tercer principio - que específicamente trata acerca del capital cooperativo - está referido, como su título indica, a la «participación económica de los socios», motivo por el cual no parecería adecuado darle cabida allí. Empero, merece destacarse que, luego de las intensas discusiones y polémicas a las que había dado lugar, la figura del socio capitalista fue finalmente acogida por vía del principio que exige salvaguardar la autonomía y la independencia de la cooperativa. Vale decir que el límite a la existencia de esa figura está dado por la necesidad de asegurar el control por parte de los socios y el mantenimiento de la autonomía cooperativa. ${ }^{10}$

La relevancia adquirida por el problema del capital en las cooperativas queda evidenciada en cuanto el Plan para una Década Coope-

9 Las leyes belga, italiana y francesa de comienzos de la década de 1990 ya habían dado cabida al socio inversor en las cooperativas, aunque la experiencia demostró que no fueron muchas las cooperativas que acudieron a esta figura. En cambio, en la misma época se produjeron importantes casos de «desmutualización» en diversos países, consistentes en que las cooperativas se transformaron en sociedades de capital y los asociados se apoderaron de las reservas acumuladas. Una muestra de la reacción crítica acerca de la incorporación de socios inversores puede verse en: Drimer, Alicia Kaplan de, "Dejaríamos entrar a los zorros en los gallineros», Cuadernos de Economía Social, n. ${ }^{\circ}$ 8-V, IAIES, Buenos Aires, septiembre 1999 y Drimer, Roberto L., «El capital en las cooperativas», Boletín de la Asociación Internacional de Derecho Cooperativo, n. ${ }^{\circ} 35$, Bilbao, 2001. En ese mismo número del Boletín se incluyen varios otros artículos sobre el tema.

10 Alianza Cooperativa Internacional, El dilema del capital en las cooperativas, Bruselas, 2016, recoge una serie de trabajos elaborados por una comisión de expertos en los que se analiza la problemática actual del capital desde diferentes ópticas y experiencias prácticas. Jean-Luis Bancel en el capítulo titulado «Capital cooperativo: una combinación indispensable de ciencia (administración) y conciencia (Principios Cooperativos)» puntualiza la importancia de los recursos propios (reservas) acumulados transgeneracionalmente, la intercooperación financiera y los inversores de capital con propósito social o filantrópico (p. 79). 
rativa aprobado por la asamblea de la $\mathrm{ACl}$, incluye al capital entre sus cinco ejes, declarando como objetivo: "Conseguir capital fiable para las cooperativas al mismo tiempo que se garantice el control por parte de los miembros» para lo cual sugiere, con carácter indicativo, una serie de acciones posibles. ${ }^{11}$

\section{El gobierno corporativo}

Estrechamente vinculados con el tema de la autonomía y la independencia se encuentran, por un lado, el gobierno corporativo en relación con la primera y, por otro, la supervisión y el control público de las cooperativas en relación con la última.

La preocupación acerca del gobierno corporativo surgió en la década de 1990 en forma prácticamente simultánea distintos países europeos (Inglaterra, Francia, España, Italia), además de los EEUU, ${ }^{12}$ incentivada posteriormente por los graves escándalos financieros que se produjeron en grandes empresas con críticas consecuencias para los accionistas, en primer lugar, pero también para los demás stakeholders. La inquietud se originó, básicamente, para asegurar a los accionistas de las sociedades cotizadas - pequeños ahorristas e inversores institucionales - la protección de sus inversiones, en cuya gestión ninguna injerencia tenían. Sin embargo, en poco tiempo el tema del buen gobierno rebasó ese ámbito y se proyectó prácticamente sobre toda clase de empresas, incluidas las propias cooperativas, sin tener en cuenta en el caso de éstas su peculiar naturaleza.

En efecto, las cooperativas no se constituyen para aumentar las inversiones realizadas por sus socios — cualquiera sea su magnitudsino para prestarles servicios que satisfagan sus necesidades de diversa clase. Por otra parte, sus socios participan democráticamente en su gobierno, por lo que éste no queda exclusivamente en manos de los accionistas mayoritarios. De allí que las motivaciones del buen gobierno

11 Alianza Cooperativa Internacional, Plan para una década cooperativa, s/ lugar de publicación, 2013, p. 32-34.

12 Son clásicos los informes de Lord Cadbury en el Reino Unido (1992), uno de los documentos más antiguos y destacados en la materia; el trabajo de la Comisión Vienot en Francia (1995); el informe Olivencia (1998) en España; la propuesta de la Comisión Draghi (1998) en Italia y los Principios de la OCDE de 1999, a partir de los cuales surgieron numerosos estudios, institutos e incluso legislaciones sobre la materia en diferentes países. EEUU merece un capítulo especial dentro del cual la Sarbanes-Oxley Act constituye expresión relevante. 
cooperativo son claramente diferentes de las que inspiran el gobierno de las corporaciones, especialmente de las sociedades cotizadas.

Por lo tanto, pretender asignar a las cooperativas el mismo esquema de gobierno que a las sociedades de capital resulta totalmente reñido con su naturaleza. La clásica dicotomía dueños/administradores se disuelve en el caso de las cooperativas pues en éstas son todos los dueños los que gobiernan en función del principio democrático y, a la vez, los que ejercen las tareas de dirección para las que son elegidos por los socios. Asimismo, los famosos «directores independientes» no tienen razón de ser en las cooperativas pues los administradores son elegidos por los propios asociados entre ellos, asegurando de esa manera que su desempeño se oriente cumplir el objetivo de la entidad, es decir satisfacer las necesidades de todos los socios y no, simplemente, asegurar la mejor rentabilidad empresaria para los mayores inversores. ${ }^{13}$

\section{Supervisión y control}

Acerca de la supervisión y el control público corresponde señalar que no se trata solamente de un problema en el caso de países con un alto grado de intromisión estatal en las cooperativas sino que suele presentarse con cierta frecuencia en países en los que la legislación no resulta invasiva pero los órganos encargados de su aplicación exceden el marco de su legítima competencia o bien exageran el celo por controlar la actuación de estas entidades. Muchas veces lo hacen en forma que sobrepasa largamente la fiscalización a la que están sujetas entidades lucrativas que realizan las mismas actividades; otras veces olvidando que las cooperativas están formadas por los propios usuarios de los servicios a los que se pretende proteger, como si los funcionarios supieran mejor que ellos sus derechos y necesidades, con total menosprecio de su capacidad y buen juicio. ${ }^{14}$

13 Para un análisis del buen gobierno cooperativo, diferente del gobierno corporativo: Cracogna, Dante; Uribe Garzón, Carlos, Buen gobierno cooperativo. Hacia un código de buenas prácticas, Confecoop, Bogotá, 2003.

14 Esta situación ha sido señalada, incluso, por organismos internacionales, como la OIT, cuya Recomendación N. ${ }^{\circ} 193$ señala que los gobiernos deberían «prever la adopción de medidas de supervisión de las cooperativas acordes con su naturaleza y funciones, que respeten su autonomía y sean conformes con la legislación y práctica nacionales y no menos favorables que las medidas aplicables a otras formas de empresa y de organización social» (parágrafo II, c). 
Este problema es diferente del que fue antes aludido al tratar acerca de la relación entre Estado y cooperativas. En efecto, la amenaza a la independencia de las cooperativas puede existir aun en los casos en los que no se considere a éstas como instrumentos de la política estatal de desarrollo puesto que la supervisión de las cooperativas en general, o de aquéllas que realizan determinadas actividades en particular, suele tener lugar en todos los países, aun en los que la política económica es indiferente hacia las cooperativas. Es decir que no se promueve o fomenta a las cooperativas, pero se las controla o fiscaliza, y en el ejercicio de esta actividad puede existir avasallamiento a su autonomía e independencia.

Lo cierto es que, en todo caso, la independencia de las cooperativas se encuentra amenazada por una acción de los gobiernos que en el ejercicio de su función de regulación o de control imponen medidas que interfieren con el libre ejercicio de sus actividades democráticamente decididas por sus asociados en el marco de la legislación general vigente, llegándose a imponer sanciones que violentan en forma manifiesta el carácter de personas jurídicas privadas de las cooperativas. A veces, la sólo existencia de esa posibilidad condiciona y limita las decisiones de estas entidades que buscan precaverse de consecuencias indeseadas.

\section{Recaudos constitucionales y legales. Caso del Mercosur}

Para concluir, cabe destacar la importancia que reviste el reconocimiento del principio de autonomía e independencia por parte de los propios Estados, traducido en disposiciones constitucionales y legales. A modo de ilustración se hará una referencia a la situación en los países del Mercosur (Argentina, Brasil, Paraguay y Uruguay) en esta materia.

Las constituciones nacionales y las leyes de cooperativas de los mencionados Estados partes del Mercosur — con excepción de la ley uruguaya 18.407 sancionada en 2008- son anteriores a la Declaración sobre la Identidad Cooperativa que incorporó de manera expresa la autonomía e independencia al plexo de los principios cooperativos. No obstante, salvo el caso de Argentina, en el que ni la Constitución reformada en 1994 ni la Ley de Cooperativas 20.337 de 1973 hacen mención del tema, en los demás países existen disposiciones constitucionales o legales que en forma manifiesta se refieren a la cuestión.

La Constitución Federal de Brasil, sancionada en 1988, al tratar sobre los derechos y deberes individuales y colectivos (art. 5. ${ }^{\circ}$ ), expresa- 
mente establece que «la creación de cooperativas, conforme a la ley, no depende de autorización, estando vedada la interferencia estatal en su funcionamiento» (par. XVIII). Esta norma tuvo efectos prácticos inmediatos pues tornó inaplicables algunas disposiciones de la Ley de Cooperativas 5764 de $1971 .{ }^{15}$ La mencionada Constitución contiene varias otras disposiciones relacionadas con las cooperativas, pero la transcripta es categórica en punto a autonomía e independencia.

El caso de Paraguay es ciertamente interesante pues allí existen tanto normas constitucionales como legales relacionadas con el principio comentado. El art. 113 de la Constitución de 1992 dispone textualmente; «El Estado fomentará la empresa cooperativa y otras formas asociativas de producción de bienes y servicios, basadas en la solidaridad y la rentabilidad social» y a continuación agrega: «a las cuales garantizará su libre organización y su autonomía.» A su vez, la Ley de Cooperativas 438 de 1994 en su art. 2. ${ }^{\circ}$ prescribe: "La libre organización y la autonomía de las cooperativas, consagradas en la Constitución Nacional, quedan garantizadas por esta ley y las disposiciones legales que en su consecuencia se dicten.» De tal suerte, Constitución y legislación recogen expresamente el principio en forma concertada. ${ }^{16}$

Por fin, en Uruguay la Constitución nada dice sobre este tema pero la Ley de Cooperativas 18.407 sancionada en 2008, es decir trece años después del Congreso del Centenario de la $\mathrm{ACl}$, dispone en su art. 2.: «El Estado garantizará y promoverá la constitución, el libre desarrollo, el fortalecimiento y la autonomía de las cooperativas, en todas sus expresiones económicas y sociales.» Por otra parte, el art. 7. ${ }^{\circ}$ establece que las cooperativas deben observar los principios cooperativos, mencionando expresamente los contenidos en la Declaración sobre la Identidad Cooperativa, señalando que dichos principios «tendrán los alcances y sentidos reconocidos por el cooperativismo universal.» Agrega el citado art. 7..$^{\circ}$ una directiva de singular relevancia como orientación para la aplicación de la ley: «Dichos principios han de aplicarse al funcionamiento y la organización de las cooperativas, han de incorpo-

15 Para los antecedentes cfr. Rose, Marco Tulio, «Cooperativismo na Constituçao: porque, quando e como», en Franke, Walmor y otros, A interferencia estatal nas cooperativas, Sergio Antonio Fabris Editor, Porto Alegre, 1985, p. 69 y ss. Para un comentario de la Constitución: Neuenschwander E. Carneiro Goldberg, Maria, «Um novo marco regulatório para o sector cooperativista brasileiro», en Guilherme Krueger (Coordenador), Cooperativas na Orden Econômica Constitucional, Tomo I, Mandamentos Editora, Belo Horizonte, 2008, p. 239 y ss.

16 Vera Díaz, Justo, Vivencias de ayuda mutua y cooperativismo en el Paraguay, Servilibro, Asunción, 2018, p. 83 y ss. 
rarse a las fuentes del derecho cooperativo como principios generales y aportan un criterio de interpretación del derecho cooperativo. ${ }^{17} \mathrm{De}$ manera que, conforme con la legislación uruguaya, existe un mandato preciso dirigido al Estado de garantizar la autonomía de las cooperativas (art. $2^{\circ}$ ) y al mismo tiempo una recepción específica de la autonomía e independencia como principio orientador de la aplicación del derecho cooperativo. Aunque no haya norma constitucional sobre la materia, como sucede en Brasil y Paraguay, sin embargo, la ley es clara en cuanto a la incorporación del principio atribuyéndole un doble carácter: mandato para el Estado y orientación para la aplicación del derecho cooperativo.

\section{Conclusión}

Los principios cooperativos constituyen un conjunto de pautas para plasmar la identidad y los valores cooperativos en la organización y el funcionamiento de las cooperativas. ${ }^{18} \mathrm{En}$ rigor, su observancia permite conocer cuándo nos hallamos frente a una genuina cooperativa, más allá de la mera denominación que ésta exhiba. En ese sentido, los principios están dirigidos, en primer lugar, a las propias cooperativas; pero también sirven de orientación para la legislación destinada a regir a estas entidades y para la actuación de los órganos del gobierno que tienen relación con las cooperativas.

El cuarto principio claramente exhibe un doble frente: autonomía al interior de la cooperativa para su funcionamiento democrático al servicio de sus miembros e independencia hacia el exterior para realizar su actividad en forma libre de condicionamientos, tanto de los gobiernos como de otras organizaciones. Desde este último punto de vista, el principio tiene una especial importancia para el trato con los gobiernos, pues representa también para éstos un límite de su actuación. ${ }^{19}$ Esta peculiar característica no se presenta en los restantes principios, los cuales están fundamentalmente dirigidos a las propiascooperativas.

17 Gutiérrez, Danilo-Lamenza, Alfredo S.-Machado, Jorge-Reyes Lavega, Sergio, Derecho cooperativo uruguayo, Fundación de Cultura Universitaria, Montevideo, 2011, p. 43 y ss.

18 Alianza Cooperativa Internacional, Notas de orientación para los principios cooperativos, $\mathrm{ACl}, \mathrm{s} / \mathrm{f}, \mathrm{p} .1$.

19 En este aspecto, los pronunciamientos de los organismos internacionales revisten notable trascendencia puesto que constituyen una directiva dirigida principalmente a los gobiernos. Cfr. Resolución ONU 56/114 y Recomendación 193 OIT. 


\section{Bibliografía}

ALIANZA COOPERATIVA INTERNACIONAL. 1987. Nuevos enfoques de los principios cooperativos en el mundo. 7a ed., Rosario: Ediciones Idelcoop.

ALIANZA COOPERATIVA INTERNACIONAL. 1996. Los principios cooperativos para el siglo XXI. Buenos Aires: Intercoop.

ALIANZA COOPERATIVA INTERNACIONAL. 2013. Plan para una década cooperativa. s/lugar de publicación.

ALIANZA COOPERATIVA INTERNACIONAL. 2015. Notas de orientación para los principios cooperativos. s/ lugar de publicación.

ALIANZA COOPERATIVA INTERNACIONAL. 2016. El dilema del capital en las cooperativas. Bruselas.

BÖÖK, Sven Ake. 1992. Valores cooperativos para un mundo en cambio. San José: ACl Oficina Regional para Centro América y el Caribe.

CRACOGNA, Dante. 1986. Estudios de Derecho Cooperativo. Buenos Aries: Intercoop.

CRACOGNA, Dante; Uribe Garzón, Carlos. 2003. Buen gobierno cooperativo. Hacia un código de buenas prácticas. Bogotá: Confecoop.

DRIMER, Alicia Kaplan de. 1999. "Dejaríamos entrar a los zorros en los gallineros», Cuadernos de Economía Social, N. ${ }^{\circ}$ 8-V, IAIES, Buenos Aires, septiembre.

DRIMER, Alicia Kaplan de; Drimer. Bernardo. 2017. Las cooperativas. Fundamentos-Historia-Doctrina. 4. ${ }^{a}$ ed. actualizada por Mirta Vuotto. Buenos Aires: Intercoop.

DRIMER, Roberto L. 2001. «El capital en las cooperativas», Boletín de la Asociación Internacional de Derecho Cooperativo, n. ${ }^{\circ}$ 35: 59-65. doi: http:// dx.doi.org/10.18543/baidc-35-2001pp59-65

GUTIÉRREZ, Danilo; Lamenza, Alfredo S.; Machado, Jorge; Reyes Lavega, Sergio. 2011. Derecho cooperativo uruguayo. Montevideo: Fundación de Cultura Universitaria.

ICA. 1980. Co-operatives and the State, London.

LAIDLAW, Alex F. s/f. Las cooperativas en el año 2000. San José: ACl Oficina Regional para Centroamérica y el Caribe.

MARCUS, Lars. s/f. Cooperativas y valores básicos. Buenos Aires: El Hogar Obrero.

MÜNKNER, Hans-H. (Editor). 2005. 100 Years Co-operative Credit Societies Act India, 1904. New Delhi: ICA Regional Office for Asia and the Pacific.

NEUENSCHWANDER E. CARNEIRO GOLDBERG, Maria. 2008. «Um novo marco regulatório para o sector cooperativista brasileiro», en Guilherme Krueger (Coordenador), Cooperativas na Orden Econômica Constitucional. Tomo I. Belo Horizonte: Mandamentos Editora.

ROSE, Marco Tulio. 1985. "Cooperativismo na Constituçao: porque, quando e como», en Franke, Walmor y otros, A interferencia estatal nas cooperativas. Porto Alegre: Sergio Antonio Fabris Editor.

URIBE GARZÓN, Carlos. 2002. Bases del cooperativismo. 5. ${ }^{a}$ ed. Bogotá: Fondo Nacional Universitario. 
VERA DÍAZ, Justo. 2018. Vivencias de ayuda mutua y cooperativismo en el Paraguay. Asunción: Servilibro.

WATKINS, W.P. 1986. Cooperative Principles Today and Tomorrow. Manchester: Holyoake Books. 


\section{Derechos de autor}

El Boletín de la Asociación Internacional de Derecho Cooperativo es una revista de acceso abierto lo que significa que es de libre acceso en su integridad inmediatamente después de la publicación de cada número. Se permite su lectura, la búsqueda, descarga, distribución y reutilización legal en cualquier tipo de soporte sólo para fines no comerciales y según lo previsto por la ley; sin la previa autorización de la Editorial (Universidad de Deusto) o el autor, siempre que la obra original sea debidamente citada (número, año, páginas y DOI si procede) y cualquier cambio en el original esté claramente indicado.

\section{Copyright}

The International Association of Cooperative Law Journal is an Open Access journal which means that it is free for full and immediate access, reading, search, download, distribution, and lawful reuse in any medium only for non-commercial purposes, without prior permission from the Publisher or the author; provided the original work is properly cited and any changes to the original are clearly indicated. 